\title{
CONJUGACY CLASSES IN ALGEBRAIC MONOIDS
}

\author{
MOHAN S. PUTCHA
}

\begin{abstract}
Let $M$ be a connected linear algebraic monoid with zero and a reductive group of units $G$. The following theorem is established.

THEOREM. There exist affine subsets $M_{1}, \ldots, M_{k}$ of $M$, reductive groups $G_{1}, \ldots, G_{k}$ with antiautomorphisms ${ }^{*}$, surjective morphisms $\theta_{i}: M_{i} \rightarrow G_{i}$, such that: (1) Every element of $M$ is conjugate to an element of some $M_{i}$, and (2) Two elements $a, b$ in $M_{i}$ are conjugate in $M$ if and only if there exists $x \in G_{i}$ such that $x \theta_{i}(a) x^{*}=\theta_{i}(b)$. As a consequence, it is shown that $M$ is a union of its inverse submonoids.
\end{abstract}

Introduction. The objects of study in this paper are connected linear algebraic monoids $M$ with zero. This means by definition that the underlying set of $M$ is an irreducible affine variety and that the product map is a morphism (i.e. a polynomial map). We will further assume that the group of units $G$ of $M$ is reductive. This means $[\mathbf{1}, \mathbf{3}]$ that the unipotent radical of $G$ is trivial. Then by $[\mathbf{6}, \mathbf{1 0}], M$ is unit regular, i.e. $M=E(M) G$ where $E=E(M)=\left\{e \in M \mid e^{2}=e\right\}$. In this paper we study the conjugacy classes of $M$. An initial study was made by the author [8], where the general problem was reduced to nilpotent elements. The approach here is quite different, yielding a more complete answer. To be precise, we show that there exist affine subsets $M_{1}, \ldots, M_{k}$ of $M$, reductive groups $G_{1}, \ldots, G_{k}$ with antiautomorphisms ${ }^{*}$, surjective morphisms $\theta_{i}: M_{i} \rightarrow G_{i}, i=1, \ldots, k$, such that: (1) Every element of $M$ is conjugate to an element of some $M_{i}$, and (2) If $a, b \in M_{i}$, then $a$ is conjugate to $b$ in $M$ if and only if there exists $x \in G_{i}$ such that $x \theta_{i}(a) x^{*}=\theta_{i}(b)$. As an application of this result, we show that $M$ is a union of its inverse submonoids. An inverse semigroup is a semigroup $S$ with the property that for each $a \in S$, there exists a unique $\bar{a} \in S$ such that $a \bar{a} a=a$ and $\bar{a} a \bar{a}=\bar{a}$. See [2]. Finally in $\S 3$, we use our main results to briefly analyze the conjugacy classes of nilpotent elements.

1. Preliminaries. Throughout this paper $Z^{+}$will denote the set of all positive integers and $K$ an algebraically closed field. Let $G$ be a connected linear algebraic group defined over $K$. The radical $R(G)$ is the maximal closed connected normal solvable subgroup of $G$ and the unipotent radical $R_{u}(G)$ is the group of unipotent elements of $R(G)$. We will assume throughout that $G$ is a reductive group, i.e., $R_{u}(G)=1$. Then $R(G) \subseteq C(G)$, the center of $G$. Moreover $G=R(G) G_{0}$ where $G_{0}=(G, G)$ is a semisimple group, i.e. $R\left(G_{0}\right)=1$. Also [3, Theorem 27.5] $G_{0}$ is a product of the simple closed normal subgroups of $G$. In particular we have the following.

Received by the editors November 3, 1986.

1980 Mathematics Subject Classification (1985 Revision). Primary 20G99, 20M10. 
FACT 1.1. If $H$ is a closed normal subgroup $G$, then $G=H C_{G}(H)$. If $H_{1}, H_{2}, H_{1}^{\prime}, H_{2}^{\prime}$ are closed normal subgroups of $G$ with $G=H_{1} H_{2}=H_{1}^{\prime} H_{2}^{\prime}$ then

$$
G=\left(H_{1} \cap H_{1}^{\prime}\right)\left(H_{1} \cap H_{2}^{\prime}\right)\left(H_{2} \cap H_{1}^{\prime}\right)\left(H_{2} \cap H_{2}^{\prime}\right) R(G) .
$$

A connected diagonalizable subgroup of $G$ is called a torus. Let $T$ be a maximal torus of $G$. Then

$$
R(G) \subseteq C(G) \subseteq C_{G}(T)=T .
$$

$W=N_{G}(T) / T$ is called the Weyl group of $G$ and is finite. A maximal closed connected solvable subgroup of $G$ is called a Borel subgroup. Let $B_{1}, B_{2}$ be Borel subgroups of $G$ with $T \subseteq B_{1} \cap B_{2}$. Then [3, Theorem 28.3] $G$ is expressible as the following disjoint union:

$$
G=\bigcup_{\sigma \in W} B_{1} \sigma B_{2}
$$

This is called the Bruhat decomposition of $G$. A subgroup of $G$ containing a Borel subgroup is called parabolic. Let $P$ be a parabolic subgroup of $G$ with $T \subseteq P$. Then there exists a parabolic subgroup $P^{-}$of $G$ such that $T \subseteq P^{-}$and $L=P \cap P^{-}$is a reductive group. $P^{-}$is called the opposite parabolic subgroup of $P$ relative to $T$ and $L$ is called a Levi factor of $P$. If $U=R_{u}(P)$, then $[\mathbf{1}, \mathbf{3}], P=L U$ is a semidirect product. This is called the Levi decomposition of $P$. By Fact 1.1, we have

FACT 1.2. Let $G=G_{1} G_{2}$ where $G_{1}, G_{2}$ are closed connected normal subgroups of $G$. Let $P$ be a parabolic subgroup of $G$. Then $P_{i}=P \cap G_{i}$ is a parabolic subgroup of $G_{i}(i=1,2)$ and $P=P_{1} P_{2}$. If $P=L U$ is a Levi decomposition of $P$, then $P_{i}=L_{i} U_{i}, L=L_{1} L_{2}, U=U_{1} U_{2}$, where $L_{i}=L \cap G_{i}, U_{i}=U \cap G_{i}, i=1,2$.

The following result follows from [1, Theorem 28.7].

FACT 1.3. Let $P, Q$ be parabolic subgroups of $G$ with Levi decompositions, $P=L_{1} U_{1}, Q=L_{2} U_{2}$ such that $T \subseteq L_{1} \cap L_{2}$. Then

$$
P \cap Q=\left(U_{1} \cap U_{2}\right)\left(U_{1} \cap L_{2}\right)\left(L_{1} \cap U_{2}\right)\left(L_{1} \cap L_{2}\right) .
$$

By a (linear) algebraic monoid, we mean a monoid $M$ such that the underlying set is an affine variety and the product map is a morphism. The identity component of $M$ is denoted by $M^{c}$. We will assume that $M$ is connected (i.e. $M=M^{c}$ ) and that $M$ has a zero. We will further asssume that the group of units $G$ of $M$ is reductive. Then by $[\mathbf{6}, \mathbf{1 0}], M$ is unit regular, i.e. $M=E(M) G$. Here $E(M)$ is the idempotent set of $M$. We fix a maximal torus $T$ of $G$. If $\Gamma \subseteq E(\bar{T})$, then we let

$$
\begin{aligned}
& C_{G}^{r}(\Gamma)=\{a \in G \mid a e=e a e \text { for all } e \in \Gamma\}, \\
& C_{G}^{l}(\Gamma)=\{a \in G \mid e a=e a e \text { for all } e \in \Gamma\} .
\end{aligned}
$$

Then $C_{G}(\Gamma)=C_{G}^{r}(\Gamma) \cap C_{G}^{l}(\Gamma)$ is a reductive group. If $e \in E(\bar{T})$, then by [5, 7], $C_{G}^{r}(e), C_{G}^{l}(e)$ are opposite parabolic subgroups of $G$. We let

$$
\begin{gathered}
G_{e}^{r}=\{a \in G \mid a e=e\}^{c}, \quad G_{e}^{l}=\{a \in G \mid e a=e\}^{c}, \\
G_{e}=\{a \in G \mid a e=e a=e\}^{c}=G_{e}^{r} \cap C_{G}(e), \\
\hat{G}_{e}=\{a \in G \mid a e=e a=e\} .
\end{gathered}
$$

Since $G_{e} \triangleleft C_{G}(e)$, we have by Fact 1.1 ,

$$
C_{G}(e)=G_{e} C_{G}\left(G_{e}\right) .
$$


In particular, $\hat{G}_{e}=G_{e} \hat{T}_{e}$. Now $e C_{G}(e)$ is the group of units of $e M e$ by [4]. In particular, $e C_{G}^{l}(e)=e C_{G}(e)$. Hence we have the surjective homomorphism: $a \rightarrow e a$ from $C_{G}^{l}(e)$ onto the reductive group $e C_{G}(e)$. Thus

$$
R_{u}\left(C_{G}^{l}(e)\right) \subseteq G_{e}^{l} \triangleleft C_{G}^{l}(e)
$$

Similarly

$$
R_{u}\left(C_{G}^{r}(e)\right) \subseteq G_{e}^{r} \triangleleft C_{G}^{r}(e) .
$$

Since $C_{G}^{r}(e)=R_{u}\left(C_{G}^{r}(e)\right) C_{G}(e)$, we get

$$
G_{e}^{r}=R_{u}\left(C_{G}^{r}(e)\right) G_{e} .
$$

LEMMA 1.4. Let $e, f \in E(\bar{T})$. Then

$$
C_{G}(e, f)=\left(G_{e} \cap G_{f}\right)\left(G_{f} \cap C_{G}\left(T_{e}\right)\right)\left(G_{e} \cap C_{G}\left(T_{f}\right)\right) C_{G}\left(T_{e}, T_{f}\right) .
$$

Proof. Now $C_{G}^{r}(f) \cap C_{G}(e)$ is a parabolic subgroup of $C_{G}(e)$ with Levi factor $C_{G}(e, f)$. Since $C_{G}(e)=G_{e} C_{G}\left(G_{e}\right)$, we have by Fact 1.2 ,

$$
C_{G}(e, f)=\left[C_{G}(f) \cap G_{e}\right]\left[C_{G}(f) \cap C_{G}\left(G_{e}\right)\right] .
$$

Similarly

$$
C_{G}(e, f)=\left[C_{G}(e) \cap G_{f}\right]\left[C_{G}(e) \cap C_{G}\left(G_{f}\right)\right] .
$$

Since $C_{G}\left(G_{e}\right) \subseteq C_{G}\left(T_{e}\right), C_{G}\left(G_{f}\right) \subseteq C_{G}\left(T_{f}\right)$, we are done by Fact 1.1 .

LEMMA 1.5. Let $e, f \in E(\bar{T})$. Then

(i) $G_{e}^{r} \cap C_{G}^{r}(f)=\left[G_{e}^{r} \cap C_{G}\left(T_{f}\right)\right]\left[G_{e}^{r} \cap G_{f}^{r}\right]$,

(ii) $G_{e}^{r} \cap C_{G}^{l}(f)=\left[G_{e}^{r} \cap C_{G}\left(T_{f}\right)\right]\left[G_{e}^{r} \cap G_{f}^{l}\right]$.

ProOF. We prove (i), as the proof of (ii) is similar. By Fact 1.3,

$$
C_{G}^{r}(e) \cap C_{G}^{r}(f)=\left[R_{u}\left(C_{G}^{r}(e)\right) \cap C_{G}^{r}(f)\right]\left[C_{G}(e) \cap C_{G}^{r}(f)\right] .
$$

Since $R_{u}\left(C_{G}^{r}(e)\right) \subseteq G_{e}^{r}$, we obtain

$$
G_{e}^{r} \cap C_{G}^{r}(f)=\left[R_{u}\left(C_{G}^{r}(e)\right) \cap C_{G}^{r}(f)\right]\left[G_{e} \cap C_{G}^{r}(f)\right] .
$$

By Facts 1.2, 1.3,

$$
\begin{aligned}
R_{u}\left(C_{G}^{r}(e)\right) \cap C_{G}^{r}(f) & =\left[R_{u}\left(C_{G}^{r}(e)\right) \cap R_{u}\left(C_{G}^{r}(f)\right)\right]\left[R_{u}\left(C_{G}^{r}(e)\right) \cap C_{G}(f)\right] \\
& \subseteq\left[G_{e}^{r} \cap G_{f}^{r}\right]\left[R_{u}\left(C_{G}^{r}(e)\right) \cap G_{f}\right]\left[R_{u}\left(C_{G}^{r}(e)\right) \cap C_{G}\left(G_{f}\right)\right] \\
& \subseteq\left[G_{e}^{r} \cap G_{f}^{r}\right]\left[G_{e}^{r} \cap C_{G}\left(T_{f}\right)\right] .
\end{aligned}
$$

Now $C_{G}(e) \cap C_{G}^{r}(f)$ is a parabolic subgroup of $C_{G}(e)$ with Levi decomposition

$$
\left[C_{G}(e, f)\right]\left[R_{u}\left(C_{G}^{r}(f)\right) \cap C_{G}(e)\right] .
$$

So by Fact 1.2 ,

By Lemma 1.4,

$$
\begin{aligned}
G_{e} \cap C_{G}^{r}(f) & =\left[G_{e} \cap C_{G}(f)\right]\left[G_{e} \cap R_{u}\left(C_{G}^{r}(f)\right)\right] \\
& \subseteq\left[G_{e} \cap C_{G}(f)\right]\left[G_{e}^{r} \cap G_{f}^{r}\right] .
\end{aligned}
$$

$$
C_{G}(e, f)=\left(C_{G}(e) \cap G_{f}\right)\left(C_{G}(e) \cap C_{G}\left(T_{f}\right)\right) .
$$

Since $G_{e} \cap C_{G}(f) \triangleleft C_{G}(e, f)$ and since the radical of $G_{e} \cap C_{G}(f)$ is contained in $T_{e} \subseteq G_{e} \cap C_{G}\left(T_{f}\right)$, we obtain

$$
G_{e} \cap C_{G}(f)=\left(G_{e} \cap G_{f}\right)\left(G_{e} \cap C_{G}\left(T_{f}\right)\right) .
$$

Since $G_{e}^{r} \cap G_{f}^{r} \triangleleft G_{e}^{r} \cap C_{G}^{r}(f)$, the result follows. 
LEMma 1.6. Let $e \in E(\bar{T})$. Then $C_{G}\left(T_{e}\right)=T_{e} C_{G}\left(G_{e}\right)$.

ProOF. Since $G_{e} \triangleleft C_{G}(e)$,

$$
C_{G}\left(G_{e}\right) \subseteq C_{G}\left(T_{e}\right) \subseteq C_{G}(e)=G_{e} C_{G}\left(G_{e}\right)
$$

So

$$
C_{G}\left(T_{e}\right)=C_{G}\left(G_{e}\right)\left[G_{e} \cap C_{G}\left(T_{e}\right)\right]=C_{G}\left(G_{e}\right) T_{e} .
$$

LEMmA 1.7. Let $e, f \in E(\bar{T})$. Then

$$
C_{G}^{r}(e) \cap C_{G}^{l}(f)=\left[G_{e}^{r} \cap C_{G}\left(T_{f}\right)\right]\left[C_{G}\left(T_{e}, T_{f}\right)\right]\left[G_{f}^{l} \cap C_{G}\left(T_{e}\right)\right]\left[G_{e}^{r} \cap G_{f}^{l}\right] .
$$

ProOF. By Fact 1.3,

$$
C_{G}^{r}(e) \cap C_{G}^{l}(f)=\left[G_{e}^{r} \cap G_{f}^{l}\right]\left[G_{e}^{r} \cap C_{G}(f)\right]\left[C_{G}(e) \cap G_{f}^{l}\right] C_{G}(e, f) .
$$

Now $G_{e}^{r} \cap G_{f}^{l} \triangleleft C_{G}^{r}(e) \cap C_{G}^{l}(f)$. Also if $a \in G_{f}^{l} \cap C_{G}\left(T_{e}\right), b \in G_{e}^{r} \cap C_{G}\left(T_{f}\right)$, then $a^{-1} b^{-1} a b \in G_{e}^{r} \cap G_{f}^{l}$. Moreover $C_{G}\left(T_{e}, T_{f}\right)$ normalizes $G_{e}^{r} \cap C_{G}\left(T_{f}\right)$ and $G_{f}^{l} \cap C_{G}\left(T_{e}\right)$. So we are done by Lemmas 1.4, 1.5.

Lemma 1.8. Let $e, f \in E(\bar{T}), a \in G_{e}^{r}, b \in C_{G}\left(T_{e}\right)$. If $a b \in C_{G}^{r}(f)$, then $a, b \in C_{G}^{r}(f)$. If $a b \in C_{G}^{l}(f)$, then $a, b \in C_{G}^{l}(f)$.

Proof. Suppose $a b \in C_{G}^{r}(f)$. Now $a=a_{1} a_{2}$ for some $a_{1} \in R_{u}\left(C_{G}^{r}(e)\right), a_{2} \in G_{e}$. Then $a_{2} b \in C_{G}(e)$. So by Fact $1.3, a_{1}, a_{2} b \in C_{G}^{r}(f)$. Then $a_{2} b \in C_{G}(e) \cap C_{G}^{r}(f)$. So by Fact 1.2, $a_{2} b=u v$ for some $u \in G_{e} \cap C_{G}^{r}(f), v \in C_{G}\left(G_{e}\right) \cap C_{G}^{r}(f)$. So $u^{-1} a_{2}=v b^{-1} \in G_{e} \cap C_{G}\left(T_{e}\right)=T_{e} \subseteq T \subseteq C_{G}^{r}(f)$. So $b \in C_{G}^{r}(f)$. Hence $a \in C_{G}^{r}(f)$.

Proposition 1.9. Let $\Gamma \subseteq E(\bar{T}), e_{1}, \ldots, e_{k+1}=f \in \Gamma$. Let $V=C_{G}(\Gamma)$, $Y_{0}=G_{f}^{l}, Y_{1}=G_{e_{1}}^{r}, Y_{i}=C_{G}\left(e_{1}, \ldots, e_{i-1}\right) \cap G_{e_{i}}^{r}$ for $i=2, \ldots, k+1$. Then

$$
Y_{0} \cdots Y_{k+1} \cap V=\prod_{i=1}^{k+1} V_{e_{i}} .
$$

PROOF. We prove by induction on $k$. So first let $k=0, a \in G_{f}^{l}, b \in G_{f}^{r}$ such that $a b \in V \subseteq C_{G}(f)$. Then $a f=a b f=f a b f=f$. So $a \in G_{f}$. Similarly $b \in G_{f}$. So

$$
a b \in G_{f} \cap V=G_{f} \cap \hat{V}_{f}=V_{f}\left(G_{f} \cap T\right)=V_{f} .
$$

So let $k>0, a \in Y_{0} \cdots Y_{k+1} \cap V$. Then $a=y_{0} \cdots y_{k+1}, y_{i} \in Y_{i}$. Now $y_{1}, \ldots, y_{k+1}$, $a \in C_{G}^{r}\left(e_{1}\right)$. Thus $y_{0} \in C_{G}^{r}\left(e_{1}\right) \cap G_{f}^{l}$. By Lemma 1.5 , there exist $\bar{y}_{0} \in G_{f}^{l} \cap$ $C_{G}\left(T_{e_{1}}\right), u \in G_{e_{1}}^{r}$ such that $y_{0}=\bar{y}_{0} u$. So $\bar{y}_{1}=u y_{1} \in G_{e_{1}}^{r}$ and $a=\bar{y}_{0} \bar{y}_{1} y_{2} \cdots y_{k+1}$. Thus without loss of generality, we may assume that $y_{0} \in C_{G}\left(T_{e_{1}}\right) \cap G_{f}^{l}$. For $i=2, \ldots, k+1$, we can factor by Lemma 1.5 ,

$$
y_{i}=c_{i} y_{i}^{\prime}, \quad c_{i} \in G_{e_{1}}, \quad y_{i}^{\prime} \in C_{G}\left(e_{1}, \ldots, e_{i-1}\right) \cap C_{G}\left(T_{e_{1}}\right) \cap G_{e_{i}}^{r} .
$$

Let

$$
d_{i}=y_{2} \cdots y_{i-1} c_{i}\left(y_{2} \cdots y_{i-1}\right)^{-1}, \quad i=3, \ldots, k+1
$$

Then

Clearly

$$
y_{1}^{\prime}=y_{1} d_{k+1} \cdots d_{3} c_{2} \in G_{e_{1}}^{r}, \quad y_{1}^{\prime \prime}=y_{0} y_{1}^{\prime} y_{0}^{-1} \in G_{e_{1}}^{r} .
$$

$$
a=y_{0} y_{1}^{\prime} y_{2}^{\prime} \cdots y_{k+1}^{\prime}=y_{1}^{\prime \prime} y_{0} y_{2}^{\prime} \cdots y_{k+1}^{\prime} .
$$


Moreover $y_{0} y_{2}^{\prime} \cdots y_{k+1}^{\prime} \in C_{G}\left(T_{e_{1}}\right)$. By Lemma 1.8, $y_{1}^{\prime \prime}, y_{0} y_{2}^{\prime} \cdots y_{k+1}^{\prime} \in V$. So $y_{1}^{\prime \prime} \in V_{e_{1}}$. By the induction hypothesis $y_{0} y_{2}^{\prime} \cdots y_{k+1}^{\prime} \in V_{e_{2}} \cdots V_{e_{k+1}}$. This completes the proof.

2. Main section. We fix a connected linear algebraic monoid $M$ with zero 0 and a reductive group of units $G$. As usual two elements $a, b \in M$ are conjugate $(a \sim b)$ if $x^{-1} a x=b$ for some $x \in G$. Note that for $a \in M, g \in G, a g \sim g a$. We fix a maximal torus $T$ of $G$. Let $W=N_{G}(T) / T$ denote the Weyl group of $G$. We let $R, \mathcal{L}, \mathcal{H}$ denote the usual Green's relations on $M[2]$. If $a, b \in M$, then $a R b$ means $a M=b M, a \mathcal{L} b$ means $M a=M b, \nLeftarrow=R \cap \mathcal{L}$. Let $e \in E(\bar{T}), \sigma=n T \in W$. Then we let

$$
e^{\sigma}=\sigma^{-1} e \sigma=n^{-1} e n \in E(\bar{T}) .
$$

We let

$$
M_{e, \sigma}=e C_{G}\left(e^{\theta} \mid \theta \in\langle\sigma\rangle\right) \sigma .
$$

Our first result is that every element of $M$ is conjugate to an element of some $M_{e, \sigma}$. In preparation, we prove

LEMMA 2.1. Let $e \in E(\bar{T}), \sigma=n T \in W, k \in Z^{+}, x, y \in C_{G}\left(e^{\sigma^{j}} \mid 0 \leq j \leq k-1\right)$, $x \in G_{e^{\sigma^{k}}}^{l}$. Then exyn $\sim$ eyn.

PROOF. We prove by induction on $k$. First let $k=1$. Then

$$
\begin{aligned}
\text { exyn } & =\text { xyen } \sim \text { yenx }=\text { yne } e^{\sigma} x \\
& =y n e^{\sigma}=\text { yen }=\text { eyn. }
\end{aligned}
$$

In general let $k>1$. Then

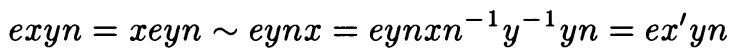

where $x^{\prime}=y_{n x n^{-1}} y^{-1} \in C_{G}\left(e^{\sigma^{j}} \mid 0 \leq j \leq k-2\right) \cap G_{e^{\sigma^{k-1}}}^{l}$. So by the induction hypothesis $e x^{\prime} y n \sim$ eyn.

THEOREM 2.2. Every element of $M$ is conjugate to an element of some $M_{e, \sigma}$.

ProOF. Let $a \in M$. By [8, Corollary 2.3], there exists a maximal torus $T_{1}$ of $G, e, f \in E\left(\bar{T}_{1}\right)$ such that $e R a \mathcal{L} f$. Since all maximal tori of $G$ are conjugate, we can assume that $T=T_{1}$. There exists $\theta=m T \in W$ such that $e^{\theta}=f$. Thus $e \operatorname{Rem} \mathcal{L} f$. So $e m \not a$. Since $e C_{G}(e)$ is the $\not{H}$-class of $e$, we see that $a \in$ $e C_{G}(e) m=e C_{G}(e) \theta$. Suppose inductively that $a \in e C_{G}\left(e^{\theta^{j}} \mid j=0, \ldots, k\right) \theta$. Let $H=C_{G}\left(e^{\theta^{j}} \mid j=0, \ldots, k\right)$. So there exists $x \in H$ such that $a=e x m$. By [5], $C_{H}^{l}\left(e^{\theta^{k+1}}\right), C_{H}^{r}\left(\theta e \theta^{-1}\right)$ are parabolic subgroups of $H$ containing $T$. By the Bruhat decomposition there exists $\pi=n_{1} T \in W(H), x_{1} \in C_{H}^{l}\left(e^{\theta^{k+1}}\right), x_{2} \in C_{H}^{r}\left(\theta e \theta^{-1}\right)$ such that $x=x_{1} n_{1} x_{2}$. So

$$
e x m=e x_{1} n_{1} x_{2} m \sim\left(m^{-1} x_{2} m\right) e x_{1} n_{1} m .
$$

Now $m^{-1} x_{2} m \in C_{G}\left(e^{\theta^{j}} \mid j=1, \ldots, k+1\right) \cap C_{G}^{r}(e)$. So

$$
m^{-1} x_{2} m e=z e \quad \text { for sorne } z \in C_{G}\left(e^{\theta^{j}} \mid j=0, \ldots, k+1\right) .
$$

Thus

$$
a \sim e z x_{1} n_{1} m, \quad z x_{1} \in C_{H}^{l}\left(e^{\theta^{k+1}}\right)
$$


Let $\lambda=\pi \theta=n_{1} m T \in W$. We claim that $e^{\lambda^{j}}=e^{\theta^{j}}$ for $j=0, \ldots, k+1$. For $j=0$, this is obvious. So assume $e^{\theta^{j}}=e^{\lambda^{j}}, j \leq k$. Then $\pi \in C_{W}\left(e^{\theta^{j}}\right)$. So

$$
e^{\lambda^{j+1}}=\left(e^{\theta^{j}}\right)^{\lambda}=\left(e^{\theta^{j}}\right)^{\pi \theta}=\left(e^{\theta^{j}}\right)^{\theta}=e^{\theta^{j+1}} .
$$

Thus $y=z x_{1} \in C_{H}^{l}\left(e^{\lambda^{k+1}}\right)$. Hence $y=y_{1} y_{2}$ for some $y_{1} \in H_{e^{\lambda^{k+1}}}^{l}, y_{2} \in C_{H}\left(e^{\lambda^{k+1}}\right)$. By Lemma 2.1,

$$
a \sim e y_{1} y_{2} n_{1} m \sim e y_{2} n_{1} m, \quad y_{2} \in C_{G}\left(e^{\lambda^{j}} \mid j=0, \ldots, k+1\right) .
$$

Continuing this process, we see that there exist $\sigma=n T \in W$ and $u \in$ $C_{G}\left(e^{\sigma^{j}}|0 \leq j \leq| W \mid\right)=C_{G}\left(e^{\gamma} \mid \gamma \in\langle\sigma\rangle\right)$ such that $a \sim$ eun. Then clearly eun $\in M_{e, \sigma}$. This completes the proof of the theorem.

Schein [13] has shown that the full transformation semigroup on any set is a union of its inverse subsemigroups. The corresponding result, for the full matrix semigroup over a field, follows from the Fitting decomposition.

THEOREM 2.3. (i) If $F$ is a commutative, idempotent submonoid of $M$, then $F N_{G}(F)$ is the maximal unit regular inverse submonoid of $M$ with idempotent set $F$.

(ii) If $F$ is a subsemilattice of $E(\bar{T})$ with $1 \in F$, then

$$
F N_{G}(F)=F C_{G}(F) N_{W}(F) .
$$

(iii) If $e \in E(\bar{T}), \sigma \in W, F=\left\langle 1, e^{\theta} \mid \theta \in\langle\sigma\rangle\right\rangle$, then $M_{e, \sigma} \subseteq F N_{G}(F)$.

(iv) $M$ is a union of its unit regular inverse submonoids.

PROOF. (i) That $F N_{G}(F)$ is a submonoid of $M$ is obvious. Let $a \in F N_{G}(F)$, $a^{2}=a$. So $a=f u$ for some $f \in F, u \in N_{G}(F)$. Then $f u f=f$. Since $M$ is a matrix semigroup and $f, u f u^{-1}$ commute, we see that $f=u f u^{-1}$. Thus $a=f u=f u f=f \in F$. So $F$ is the idempotent set of $F N_{G}(F)$. It follows that $F N_{G}(F)$ is the maximal unit regular submonoid of $M$ with idempotent set $F$. Since $F$ is commutative, it follows [2] that $F N_{G}(F)$ is an inverse semigroup.

(ii) Let $a \in N_{G}(F)$. Clearly $T \subseteq C_{G}(F)$. So $a T a^{-1} \subseteq C_{G}\left(a F a^{-1}\right)=C_{G}(F)$. So $T, a T a^{-1}$ are maximal tori of $C_{G}(F)$. Hence $b^{-1} a T a^{-1} b=T$ for some $b \in C_{G}(F)$. Hence $b^{-1} a \in N_{G}(T) \cap N_{G}(F)$. So $a=b\left(b^{-1} a\right) \in C_{G}(F) N_{W}(F)$.

(iii), (iv) follow from (ii) and Theorem 2.2 .

Now fix $e \in E(\bar{T}), \sigma=n T \in W$. Let $f=e^{\sigma}, \alpha+1$ the order of $\sigma$. Let

$$
V=C_{G}\left(e^{\theta} \mid \theta \in\langle\sigma\rangle\right) \text {. }
$$

So $V$ is a reductive group, $T \subseteq V, V^{\sigma}=V, M_{e, \sigma}=e V \sigma$. Now $\hat{V}_{e}=\{a \in V \mid a e=$ $e a=e\}=\hat{T}_{e} V_{e}$ is a closed normal subgroup of $V$. Let

$$
\Omega=\prod_{\theta \in\langle\sigma\rangle} \hat{V}_{e}^{\theta}=\prod_{\theta \in\langle\sigma\rangle} \hat{V}_{e^{\theta}}
$$

Then $\Omega$ is a closed normal subgroup of $V$. If $x \in V$, let $x^{*}=n x^{-1} n^{-1} \in V$. Then $\Omega^{*}=\Omega$. So ${ }^{*}$ induces an antiautomorphism * on the reductive group $G_{e, \sigma}=V / \Omega$. Define $\xi: M_{e, \sigma} \rightarrow G_{e, \sigma}$ as follows: If $a=e v n \in M_{e, \sigma}, v \in V$, then, $\xi(a)=v \Omega \in$ $G_{e, \sigma}$. Since $\hat{V}_{e} \subseteq \Omega, \xi$ is well defined. Note further that if $G_{e, \sigma}$ is replaced by $e V / e \Omega$ (which is isomorphic to $G_{e, \sigma}$ as an abstract group), then $\xi$ would also be a morphism of varieties. 
THEOREM 2.4. Let $a, b \in M_{e, \sigma}$. Then $a$ is conjugate to $b$ in $M$ if and only if there exists $x \in G_{e, \sigma}$ such that $x \xi(a) x^{*}=\xi(b)$.

PROOF. For $a, b \in M_{e, \sigma}$, define $a \equiv b$ if $x \xi(a) x^{*}=\xi(b)$ for some $x \in G_{e, \sigma}$. We are to show that $\equiv=\sim$. Let

$$
A=\{a \in V \mid \text { eun } \sim \text { eaun for all } u \in V\} .
$$

Clearly $\hat{V}_{e} \subseteq A$. Let $a, b \in A$. Then for $u \in V$, eabun $\sim$ ebun $\sim$ eun. So $A^{2} \subseteq A$. Now let $a \in A, u \in V$. Then

$$
\begin{aligned}
e\left(\text { nan }^{-1}\right) u n & \sim a n^{-1} \text { unen }=e a\left(n^{-1} \text { un }\right) n \\
& \sim e\left(n^{-1} \text { un }\right) n=n^{-1} \text { unen } \sim \text { eun } .
\end{aligned}
$$

Thus $n A n^{-1} \subseteq A$. It follows that $\Omega \subseteq A$. Now let $m_{1}, m_{2} \in M_{e, \sigma}$ such that $m_{1} \equiv m_{2}$. Let $m_{1}=$ eun, $m_{2}=$ evn where $u, v \in V$. Then there exists $x \in V$ such that $v \in \Omega$ xunx $x^{-1} n^{-1}$. Since $\Omega \subseteq A$,

$$
\begin{aligned}
m_{1} & =\text { evn } \sim \text { exunx } x^{-1} n^{-1} n=e x u n x^{-1} \\
& =\text { eeunx } x^{-1} \sim \text { eun }=m_{2} .
\end{aligned}
$$

This shows that $\equiv \subseteq \sim$.

Conversely let $m_{1}, m_{2} \in M_{e, \sigma}$ such that $m_{1} \sim m_{2}$. Then there exists $X_{1} \in G$ such that

$$
X_{1} m_{1}=m_{2} X_{1} \text {. }
$$

Let $m_{1}=e u n, m_{2}=e v n$ where $u, v \in V$. Then by (1),

$$
X_{1} e R X_{1} \text { eun }=e v n X_{1} R e
$$

So $X_{1} e=e X_{1} e$ and $X_{1} \in C_{G}^{r}(e)$. Also by (1),

$$
f X_{1}=n^{-1} e n X_{1} \mathcal{L} m_{2} X_{1}=X_{1} m_{1} \mathcal{L} m_{1} \mathcal{L} f \text {. }
$$

Thus $X_{1} \in C_{G}^{r}(e) \cap C_{G}^{l}(f)$. By Lemma 1.7, $X_{1} \in X\left[G_{e}^{r} \cap G_{f}^{l}\right]$ for some

$$
X \in\left[C_{G}\left(T_{e}\right) \cap G_{f}^{l}\right]\left[C_{G}\left(T_{e}, T_{f}\right)\right]\left[C_{G}\left(T_{f}\right) \cap G_{e}^{r}\right] .
$$

Since $m_{1}=e m_{1}, m_{2}=m_{2} f$, we see by (1) that

$$
X e u n=e v n X .
$$

Now $X=a x b$ for some

$$
a \in C_{G}\left(T_{e}\right) \cap G_{f}^{l}, \quad x \in C_{G}\left(T_{e}, T_{f}\right), \quad b \in C_{G}\left(T_{f}\right) \cap G_{e}^{r} .
$$

So by (2), eaxun $=e v n x b$. Then $e a x u=e v n x b n^{-1}$ and $a x u, v n x b n^{-1} \in C_{G}(e)$. So

$$
a x u=v n x b n^{-1} z \quad \text { for some } z \in \hat{G}_{e} .
$$

Now $n x b n^{-1} \in C_{G}\left(T_{e}\right)$. So by Lemma $1.6, n x b n^{-1}=t \eta$ for some $t \in T, \eta \in$ $C_{G}\left(G_{e}\right)$. So $v t \in V \subseteq C_{G}(e)$. So $v t=v^{\prime} v^{\prime \prime}$ for some $v^{\prime} \in C_{V}\left(V_{e}\right) \subseteq C_{G}\left(T_{e}\right)$, $v^{\prime \prime} \in V_{e} \subseteq G_{e}$. Also $u=u^{\prime} u^{\prime \prime}$ for some $u^{\prime} \in C_{V}\left(V_{e}\right) \subseteq C_{G}\left(T_{e}\right), u^{\prime \prime} \in V_{e} \subseteq G_{e}$. Then

$$
a x u=v n x b n^{-1} z=v t \eta z=v^{\prime} v^{\prime \prime} \eta z=v^{\prime} \eta v^{\prime \prime} z .
$$

So

$$
a x u^{\prime}=v^{\prime} \eta\left(v^{\prime \prime} z\left(u^{\prime \prime}\right)^{-1}\right)
$$


Let $z^{\prime}=v^{\prime \prime} z\left(u^{\prime \prime}\right)^{-1}$. Then $z^{\prime} \in \hat{G}_{e}$. So $z^{\prime} h=h z^{\prime}=h$ for all $h \in E(M)$ with $h \leq e$. Now $a x u^{\prime}, v^{\prime}, \eta \in C_{G}\left(T_{e}\right)$. So by $(5), z^{\prime} \in C_{G}\left(T_{e}\right)$. Thus $z^{\prime} h=h z^{\prime}$ for all $h \in E(\bar{T})$ with $h \geq e$. So for any maximal chain $\Gamma$ of $E(\bar{T})$ with $e \in \Gamma, z^{\prime} \in C_{G}(\Gamma)=T \subseteq V$. Let $u_{1}=u^{\prime}\left(z^{\prime}\right)^{-1} v^{\prime \prime} \in V$. Then by (5),

$$
a x u_{1}=v^{\prime} \eta v^{\prime \prime}=v^{\prime} v^{\prime \prime} \eta=v t \eta=v n x b n^{-1} .
$$

Also $z=\left(v^{\prime \prime}\right)^{-1} z^{\prime} u^{\prime \prime} \in V \cap \hat{G}_{e}=\hat{V}_{e}$. So

$$
a x u_{1}=v n x b n^{-1}, \quad u_{1}, v \in V, z \in \hat{V}_{e} .
$$

Now $x b \in C_{G}^{r}(e)$. So $n x b n^{-1} \in C_{G}^{r}\left(\sigma e \sigma^{-1}\right)$. Thus $a x \in C_{G}^{r}\left(\sigma e \sigma^{-1}\right)$. By (3), Lemma $1.8, a, x \in C_{G}^{r}\left(\sigma e \sigma^{-1}\right)$. So $x \in C_{G}^{r}\left(\sigma e \sigma^{-1}\right) \cap C_{G}\left(T_{e}, T_{f}\right)$. Hence we can factor

(7) $x=y_{1} x_{1} \quad$ for some $y_{1} \in G_{\sigma e \sigma^{-1}}^{r} \cap C_{G}\left(T_{e}, T_{f}\right), x_{1} \in C_{G}\left(T_{\sigma e \sigma^{-1}}, T_{e}, T_{f}\right)$.

Also $a \in C_{G}^{r}\left(\sigma e \sigma^{-1}\right) \cap C_{G}\left(T_{e}\right) \cap G_{f}^{l}$. So working within $C_{G}\left(T_{e}\right)$ and applying Lemma 1.5 , we can factor

(8) $a=c_{1} a_{1} \quad$ for some $c_{1} \in G_{\sigma e \sigma^{-1}}^{r} \cap G_{f}^{l} \cap C_{G}\left(T_{e}\right), a_{1} \in C_{G}\left(T_{e}, T_{\sigma e \sigma^{-1}}\right) \cap G_{f}^{l}$.

Now by (6),

$$
c_{1} a_{1} y_{1} x_{1} u_{1}=v n y_{1} x_{1} b n^{-1}
$$

So

$$
w a_{1} x_{1} u_{1}=v n y_{1} x_{1} n^{-1}
$$

where

$$
w=c_{1} a_{1} y_{1} a_{1}^{-1}\left[\left(a_{1} x_{1} u_{1}\right)\left(n b^{-1} n^{-1}\right)\left(a_{1} x_{1} u_{1}\right)^{-1}\right] \in G_{\sigma e \sigma^{-1}}^{r} .
$$

Suppose now inductively that

$$
x=y_{1} \cdots y_{k} x_{k}
$$

where

$$
\begin{aligned}
y_{i} & \in C_{G}\left(T_{f}, T_{\sigma^{j} e \sigma^{-1}} \mid j=0, \ldots, i-1\right) \cap G_{\sigma^{i} e \sigma^{-i}}^{r}, \quad i=1, \ldots, k, \\
x_{k} & \in C_{G}\left(T_{f}, T_{\sigma^{j} e \sigma^{-j}} \mid j=0, \ldots, k\right) .
\end{aligned}
$$

Further assume that there exist

$$
\begin{aligned}
& w_{i} \in C_{G}\left(T_{\sigma^{j} e \sigma^{-j}} \mid i+1 \leq j \leq k\right) \cap G_{\sigma^{i} e \sigma^{-i}}^{r}, \quad i=1, \ldots, k, \\
& a_{k} \in C_{G}\left(T_{\sigma^{i} e \sigma^{-i}} \mid i=0, \ldots, k\right) \cap G_{f}^{l}
\end{aligned}
$$

such that

$$
w_{k} \cdots w_{1} a_{k} x_{k} u_{1}=v n y_{k} x_{k} n^{-1}
$$

Note that (7)-(9) show (10)-(12) to be valid for $k=1$. Now

$$
n y_{k} x_{k} n^{-1} \in C_{G}^{r}\left(\sigma^{k+1} e \sigma^{-k-1}\right) \text {. }
$$

So by (12), $w_{k} \cdots w_{1} a_{1} x_{k} \in C_{G}^{r}\left(\sigma^{k+1} e \sigma^{-k-1}\right)$. Repeated use of Lemma 1.8 shows that $w_{1}, \ldots, w_{k}, a_{k}, x_{k} \in C_{G}^{r}\left(\sigma^{k+1} e \sigma^{-k-1}\right)$. So by Lemma 1.5 , we can factor for 
$i=1, \ldots, k$,

$$
\begin{array}{ll}
w_{i}=q_{i} w_{i}^{\prime}, & w_{i}^{\prime} \in C_{G}\left(T_{\sigma^{j} e \sigma^{-j}} \mid i+1 \leq j \leq k+1\right) \cap G_{\sigma^{i} e \sigma^{-i}}^{r}, \\
& q_{i} \in G_{\sigma^{k+1} e \sigma^{-k-1}}^{r} \cap C_{G}\left(T_{\sigma^{j} e \sigma^{-j}} \mid i+1 \leq j \leq k\right), \\
a_{k}=c_{k+1} a_{k+1}, & a_{k+1} \in C_{G}\left(T_{\sigma^{j} e \sigma^{-j}} \mid 0 \leq j \leq k+1\right) \cap G_{f}^{l}, \\
& c_{k+1} \in C_{G}\left(T_{\sigma^{j} e \sigma^{-j}} \mid 0 \leq j \leq k\right) \cap G_{\sigma^{k+1} e \sigma^{-k-1}}^{r}, \\
x_{k}=y_{k+1} x_{k+1}, & x_{k+1} \in C_{G}\left(T_{f}, T_{\sigma^{j} e \sigma^{-j}} \mid 0 \leq j \leq k+1\right), \\
& y_{k+1} \in C_{G}\left(T_{f}, T_{\sigma^{j} e \sigma^{-j}} \mid 0 \leq j \leq k\right) \cap G_{\sigma^{k+1} e \sigma^{-k-1}}^{r} .
\end{array}
$$

Let

$$
\begin{aligned}
q_{i}^{\prime} & =w_{k}^{\prime} \cdots w_{i+1}^{\prime} q_{i}\left(w_{k}^{\prime} \cdots w_{i+1}^{\prime}\right)^{-1} \in G_{\sigma^{k+1}}^{r} e \sigma^{-k-1} \\
c_{k+1}^{\prime} & =w_{k}^{\prime} \cdots w_{1}^{\prime} c_{k+1}\left(w_{k}^{\prime} \cdots w_{1}^{\prime}\right)^{-1} \in G_{\sigma^{k+1}}^{r} e \sigma^{-k-1} \\
y_{k+1}^{\prime} & =w_{k}^{\prime} \cdots w_{1}^{\prime} a_{k+1} y_{k+1}\left(w_{k}^{\prime} \cdots w_{1}^{\prime} a_{k+1}\right)^{-1} \in G_{\sigma^{k+1}}^{r} e \sigma^{-k-1} \\
p & =q_{k} q_{k-1}^{\prime} \cdots q_{1}^{\prime} c_{k+1}^{\prime} y_{k+1}^{\prime} \in G_{\sigma^{k+1}}^{r} e \sigma^{-k-1}
\end{aligned}
$$

Then

So by (12),

$$
w_{k} \cdots w_{1} a_{k} x_{k}=p w_{k}^{\prime} \cdots w_{1}^{\prime} a_{k+1} x_{k+1}
$$

where

$$
w_{k+1}^{\prime} \cdots w_{1}^{\prime} a_{k+1} x_{k+1} u_{1}=v n y_{k+1} x_{k+1} n^{-1}
$$

$$
w_{k+1}^{\prime}=v n y_{k}^{-1} n^{-1} v^{-1} p \in G_{\sigma^{k+1}}^{r} e \sigma^{-k-1} .
$$

This completes the induction step in (10)-(12). So (10) is valid for all $k \in Z^{+}$. In particular it is valid for $k=\alpha$, where $\sigma^{\alpha+1}=1$. Then

$$
x=y_{1} \cdots y_{\alpha} x_{\alpha}, \quad x_{\alpha} \in C_{G}\left(e^{\theta} \mid \theta \in\langle\sigma\rangle\right)=V .
$$

Now by (4), (6)

$$
a x u=v n x b n^{-1} z, \quad z \in \hat{V}_{e}
$$

Let $Y_{0}=G_{f}^{l}, Y_{1}=G_{\sigma e \sigma^{-1}}^{r}$,

$$
Y_{i}=C_{G}\left(\sigma^{j} e \sigma^{-j} \mid j=1, \ldots, i-1\right) \cap G_{\sigma^{i} e \sigma^{-i}}^{r}, \quad i \geq 2 .
$$

Then $Y_{j}$ normalizes $Y_{i}$ for $j \geq i \geq 1$. Also $V$ normalizes $Y_{i}$ for all $i$. By (3), (11) we see that

$$
a \in Y_{0}, \quad n b n^{-1} \in Y_{1}, \quad y_{i} \in Y_{i}, \quad 1=1, \ldots, \alpha .
$$

Also, since $\sigma^{\alpha+1}=1$, we see by (11) that

$$
n y_{i} n^{-1} \in Y_{i+1}, \quad i=1, \ldots, \alpha-1, \quad n y_{\alpha} n^{-1} \in V_{e} .
$$

Since $x_{\alpha}, u, v \in V$ and $V^{\sigma}=V$, we see by (13)-(16),

$$
\begin{aligned}
v\left(x_{\alpha} u x_{\alpha}^{*}\right)^{-1}= & v n x_{\alpha} n^{-1} u^{-1} x_{\alpha}^{-1} \\
= & a x u z^{-1} n b^{-1} x^{-1} x_{\alpha} n^{-1} u^{-1} x_{\alpha}^{-1} \\
= & {\left[a y_{1} \cdots y_{\alpha} x_{\alpha} u z^{-1} u^{-1} x_{\alpha}^{-1}\right]\left(x_{\alpha} u\right) } \\
& \times\left[n b^{-1} x_{\alpha}^{-1}\left(y_{\alpha}^{-1} \cdots y_{1}^{-1}\right) x_{\alpha} n^{-1}\right]\left(x_{\alpha} u\right)^{-1} \\
\in & \hat{V}_{e} Y_{0} Y_{1} \cdots Y_{\alpha} .
\end{aligned}
$$

Since $\sigma^{\alpha} e \sigma^{-\alpha}=f$, we see by Proposition 1.9 that $v\left(x_{\alpha} u x_{\alpha}^{*}\right)^{-1} \in \Omega$. Thus $m_{1}=$ eun $\equiv$ evn $=m_{2}$. This completes the proof of the theorem. 
The proof of the above theorem shows

COROllaRy 2.5. Let $a, b \in M_{e, \sigma}$. Then $a \sim b$ if and only if there exists $x \in V=C_{G}\left(e^{\theta} \mid \theta \in\langle\sigma\rangle\right)$ such that $x^{-1} a x=b$.

COROllary 2.6. Let $D=e C_{G}(e)$ denote the group of units of eMe, $h \in$ $E(\overline{e T}), \theta=m T \in C_{W}(e)$. Then $M_{h, \theta}=(e M e)_{h, e \theta}$ and $G_{h, \theta} \cong D_{h, e \theta}$. If $a, b \in$ $M_{h, \theta}$, then $a$ is conjugate to $b$ in $M$ if and only if $a$ is conjugate to $b$ in eMe.

PROOF. Let

$$
V=C_{G}\left(h^{\gamma} \mid \gamma \in\langle\theta\rangle\right), \quad Y=C_{D}\left(h^{\gamma} \mid \gamma \in\langle\theta\rangle\right) .
$$

Let $a \in Y$. Then $a=e x$ for some $x \in C_{G}(e)$. For $\gamma \in\langle\theta\rangle$,

$$
x h^{\gamma}=x e h^{\gamma}=a h^{\gamma}=h^{\gamma} a=h^{\gamma} e x=h^{\gamma} x .
$$

So $x \in C_{V}(e)$ and $Y=e C_{V}(e)$. Now $V=V_{h} C_{V}\left(V_{h}\right)=V_{h} C_{V}(e)$. Hence

$$
M_{h, \theta}=h V \theta=h C_{V}(e) \theta=h e C_{V}(e) \theta=h Y \theta=(e M e)_{h, e \theta} .
$$

Let $\Omega=\prod_{\gamma \in\langle\theta\rangle} \hat{V}_{h^{\gamma}}$. Since $V=V_{h} C_{V}(e), h \leq e$,

$$
G_{h, \theta}=V / \Omega \cong C_{V}(e) / C_{\Omega}(e) \cong e V / e C_{\Omega}(e) .
$$

By Proposition 1.9,

$$
C_{\Omega}(e)=\prod_{\gamma \in\langle\theta\rangle}\left[\hat{V}_{h^{\gamma}} \cap C_{G}(e)\right] .
$$

It follows that $e V / e C_{\Omega}(e)=D_{h, e \theta}$. We are now done by Theorem 2.4 .

CONJECTURE 2.7. Let $a, b \in e M e$. Then $a$ is conjugate to $b$ in $M$ if and only if $a$ is conjugate to $b$ in $e M e$.

ConjeCture 2.8. Let $\mathcal{y}=\left\{M_{e, \sigma} \mid e \in E(\bar{T}), \sigma \in W\right\}, y_{0}$ the set of maximal elements (with respect to inclusion) of $\mathcal{Y}$. Then if $Y_{1}, Y_{2} \in \mathcal{Y}_{0}, a \in Y_{1}, b \in Y_{2}$, $a \sim b$, then $Y_{1}^{\theta}=Y_{2}$ for some $\theta \in W$.

Let $g \in G$. Then the map: $x \rightarrow g x^{-1} g^{-1}$ is an antiautomorphism of $G$. We will call such an antiautomorphism an inner antiautomorphism.

EXAMPLE 2.9. Let $n \in Z^{+}, M=\mathcal{M}_{n}(K)$. Let $h=\prod_{\theta \in\langle\sigma\rangle} e^{\theta}, r$ the rank of $h$. Then $G_{e, \sigma} \cong \mathrm{GL}(r, K)$ and ${ }^{*}$ is an inner antiautomorphism.

EXAMPLE 2.10. Let $M=\left\{A \otimes B \mid A, B \in \mathcal{M}_{2}(K)\right\}$. Then the possibilities for $G_{e, \sigma}$ are $G, \mathrm{SL}(2, K), \operatorname{PGL}(2, K), G_{m},\{1\}$. In all cases, * is inner.

CONJECTURE 2.11. If the simple components of $G$ are all of type $A_{l}$, then ${ }^{*}$ is necessarily inner.

By [3, Theorem 27.4], an antiautomorphism of a semisimple group is the composition of an inner antiautomorphism and an automorphism determined by an automorphism of the Dynkin diagram of the group.

CONJECTURE 2.12. For all $t \in R\left(G_{e, \sigma}\right), t^{*}=t^{-1}$ and hence ${ }^{*}$ is completely determined by its action on the semisimple group $G_{e, \sigma}^{\prime}=\left(G_{e, \sigma}, G_{e, \sigma}\right)$.

3. Nilpotent elements. We continue from [8] the analysis of conjugacy classes of nilpotent elements of $M$. It was shown in [8] that the conjugacy classes of minimal nilpotent elements (in the $J$-class ordering) is always finite. Renner [12] has introduced the finite fundamental inverse monoid $\operatorname{Ren}(M)=N_{G}(\bar{T}) / T$ and 
used it to generalize the Bruhat decomposition to $M$. We easily have

Proposition 3.1. Let $e \in E(\bar{T}), \sigma=n T \in W, k \in Z^{+}$. Then the following conditions are equivalent:

(i) $a^{k}=0$ for some $a \in M_{e, \sigma}$,

(ii) $M_{e, \sigma}^{k}=0$,

(iii) $(e \sigma)^{k}=0$ in $\operatorname{Ren}(M)$,

(iv) $e^{\sigma} \cdots e^{\sigma^{k}}=0$.

Since $V=C_{G}\left(e^{\theta} \mid \theta \in\langle\sigma\rangle\right)$ is a reductive group, we see that any closed normal subgroup of $V$ containing $T$, must equal $V$. Thus

Proposition 3.2. Let $e \in E(\bar{T}), \sigma \in W$. Then $G_{e, \sigma}$ is trivial if and only if $T=\prod_{\theta \in\langle\sigma\rangle} T_{e^{\theta}}$.

In particular, we see that $G_{e, \sigma}$ trivial implies that $e \sigma$ is nilpotent. If the groups $G_{e, \sigma}$ are trivial for all nilpotent $e \sigma$, then by Theorems 2.2, 2.4, the number of conjugacy classes of nilpotent elements in $M$ is finite.

CONJECTURE 3.3. The number of conjugacy classes of nilpotent elements of $M$ is finite if and only if the groups $G_{e, \sigma}$ are trivial for all nilpotent $e \sigma$.

EXAMPLE 3.4. If $M=\mathcal{M}_{n}(K)$, then we see by Example 2.9 that the groups $G_{e, \sigma}$ are trivial for nilpotent $e \sigma$.

EXAMPLE 3.5. Let $G_{0}=\left\{A \otimes\left(A^{-1}\right)^{t} \mid A \in \mathrm{SL}(3, K)\right\}, G=K^{*} G_{0}, M=\overline{K G}_{0}$. Let $S=M \backslash G$. Then

$$
E(S)=\left\{e \otimes f \mid e^{2}=e, f^{2}=f \in \mathcal{M}_{3}(K), e f^{t}=f^{t} e=0\right\} .
$$

In particular

$$
e=\left[\begin{array}{lll}
1 & 0 & 0 \\
0 & 0 & 0 \\
0 & 0 & 0
\end{array}\right] \otimes\left[\begin{array}{lll}
0 & 0 & 0 \\
0 & 1 & 0 \\
0 & 0 & 1
\end{array}\right], \quad f=\left[\begin{array}{lll}
0 & 0 & 0 \\
0 & 1 & 0 \\
0 & 0 & 0
\end{array}\right] \otimes\left[\begin{array}{lll}
1 & 0 & 0 \\
0 & 0 & 0 \\
0 & 0 & 1
\end{array}\right] \in E(M)
$$

Also if

$$
\sigma=\left[\begin{array}{ccc}
0 & 1 & 0 \\
1 & 0 & 0 \\
0 & 0 & -1
\end{array}\right] \otimes\left[\begin{array}{ccc}
0 & 1 & 0 \\
1 & 0 & 0 \\
0 & 0 & -1
\end{array}\right] \in W(G)
$$

then $e^{\sigma}=f$ and $(e \sigma)^{2}=0$. The group $G_{e, \sigma}$ can be seen to be the one dimensional torus with ${ }^{*}$ being given by $x \rightarrow x^{-1}$. Thus by Theorem 2.4 , the number of conjugacy classes of nilpotent elements of $M$ is infinite. However if $C$ denotes the center of $G$, then the number of conjugacy classes of nilpotent elements in $M / C$ is finite.

EXAMPLE 3.6. Suppose char $K \neq 2, n \in Z^{+}, n \geq 2$. For $r \in Z^{+}$, let $J_{r}$ denote the $r \times r$ matrix

$$
\left[\begin{array}{lll} 
& & 1 \\
1 & \cdot & \\
& &
\end{array}\right]
$$

Let $G_{0}$ consist of all $A \in \mathrm{SL}(2 n+1, K)$ such that

$$
A^{t}\left[\begin{array}{cc}
1 & 0 \\
0 & J_{2 n}
\end{array}\right] A=\left[\begin{array}{cc}
1 & 0 \\
0 & J_{2 n}
\end{array}\right] \text {. }
$$


Thus $[3, \S 7.2], G_{0}$ is the special orthogonal group of type $B_{n}$. Let $G=K^{*} G_{0}$, $M=\overline{K G}_{0}$. Then

$$
e=\left[\begin{array}{ccc}
0 & 0 & 0 \\
0 & I_{n} & 0 \\
0 & 0 & 0
\end{array}\right], \quad f=\left[\begin{array}{ccc}
0 & 0 & 0 \\
0 & 0 & 0 \\
0 & 0 & I_{n}
\end{array}\right] \in E(M) .
$$

If

$$
\sigma=\left[\begin{array}{cc} 
\pm 1 & 0 \\
0 & J_{2 n}
\end{array}\right] \in W(G)
$$

then $e^{\sigma}=f$ and $(e \sigma)^{2}=0$. It can be seen that $G_{e, \sigma} \cong \operatorname{PGL}(n, K)$ with the antiautomorphism ${ }^{*}$ on $G_{e, \sigma}$ given by $A \rightarrow J_{n} A^{t} J_{n}$. Thus by Theorem 2.4, the number of conjugacy classes of nilpotent elements of $M$ is infinite. This gives a counterexample to $[8$, Conjectures $4.5,4.6]$. Note also that for $n \geq 3,{ }^{*}$ is not inner.

The above examples suggest

CONJECTURE 3.7. Suppose that the center of $G$ is one dimensional. Then the number of conjugacy classes of nilpotent elements of $M$ is finite if and only if $\operatorname{Ren}(M)$ is isomorphic to the symmetric inverse semigroup of some finite set.

\section{REFERENCES}

1. R. W. Carter, Finite groups of Lie type: Conjugacy classes and complex characters, Wiley, 1985.

2. J. M. Howie, An introduction to semigroup theory, Academic Press, 1976.

3. J. E. Humphreys, Linear algebraic groups, Springer-Verlag, 1981.

4. M. S. Putcha, Green's relations on a connected algebraic monoid, Linear and Multilinear Algebra 12 (1982), 205-214.

5. __ A semigroup approach to linear algebraic groups, J. Algebra 80 (1983), 164-185.

6. __ Reductive groups and regular semigroups, Semigroup Forum 30 (1984), 253-261.

7. __ Determinant functions on algebraic monoids, Comm. Algebra 11 (1983), 695-710.

8. __ Regular linear algebraic monoids, Trans. Amer. Math. Soc. 290 (1985), 615-626.

9. L_ Linear algebraic monoids, monograph (to appear).

10. L. E. Renner, Reductive monoids are von Neumann regular, J. Algebra 93 (1985), 237-245.

11. _ Classification of semisimple algebraic monoids, Trans. Amer. Math. Soc. 292 (1985), 193-224.

12. __ Analogue of the Bruhat decomposition for algebraic monoids, J. Algebra 101 (1986), 303-338.

13. B. M. Schein, A symmetric semigroup of transformations is covered by its inverse subsemigroups, Acta Math. Acad. Sci. Hur.gar. 22 (1971), 163-171.

Department of Mathematics, North Carolina State University, Raleigh, NORTH CAROLINA 27695-8205 